Quim. Nova, Vol. 34, No. 1, 76-81, 2011

\title{
SÍNTESE E AVALIAÇÃO PRELIMINAR DA ATIVIDADE ANTINOCICEPTIVA DE NOVAS ISOXAZOLIL- ARIL-HIDRAZONAS
}

Sílvio Leandro Gonçalves Bomfim Reis, Valderes Moraes de Almeida, Gleybson Correia de Almeida, Karinna Moura Boaviagem, Charles Christophe Du Barriere Mendes e Antônio Rodolfo de Faria*

Departamento de Ciências Farmacêuticas, Universidade Federal de Pernambuco, Cidade Universitária, 50740-521 Recife - PE, Brasil Alexandre José da Silva Góes, Laudelina Rodrigues Magalhães e Teresinha Gonçalves da Silva

Departamento de Antibióticos, Universidade Federal de Pernambuco, Cidade Universitária, 50670-901 Recife - PE, Brasil

Recebido em 12/2/10; aceito em 6/7/10; publicado na web em 16/11/10

\begin{abstract}
SYNTHESIS AND PRELIMINARY EVALUATION OF ANTINOCICEPTIVE ACTIVITY OF NOVEL ISOXAZOLYL-ARYLHYDRAZONES. New 2-isoxazoline aldehydes were synthesized, in good yields, from cycloadduct of the 1,3-dipolar cycloaddition reaction between endocyclic enecarbamate and carboethoxyformonitrile oxide (CEFNO). Condensation of these 2-isoxazoline aldehydes with several phenyl-hydrazines produced new isoxazolyl-aryl-hydrazones, which showed low toxicity and excellent antinociceptive activity, when compared to dipyrone. The antinociceptive activity of isoxazolyl-aryl-hydrazones was performed using the acetic acid-induced mice abdominal constrictions test
\end{abstract}

Keywords: 2-isoxazoline aldehydes; hydrazones; antinociceptive activity.

\section{INTRODUÇÃO}

Ciclo-oxigenase (COX) é a enzima chave envolvida na biossíntese de prostanoides (prostaglandinas, prostaciclinas e tromboxanos), que são substâncias envolvidas em diversos processos fisiológicos, assim como em processos patológicos, como a inflamação. ${ }^{1}$ Duas isoformas de COX são identificadas, uma forma constitutiva, conhecida como COX-1, e uma forma indutível, conhecida como COX-2, além da COX-3, uma variante da COX-1. Estas enzimas são sensíveis à inibição por substâncias anti-inflamatórias não esteroidais (AINEs).

Parece evidente que COX-1 desempenha um papel chave na homeostase, enquanto que a COX-2 atua em condições patológicas como inflamação ou mesmo proliferação de câncer.

AINEs clássicos agem como inibidores não seletivos da COX, sendo utilizados como anti-inflamatórios, analgésicos e antipiréticos, principalmente para tratar a inflamação crônica. No entanto, AINEs não seletivos podem causar efeitos colaterais relacionados com a inibição da COX-1 como, por exemplo, a irritação gastrointestinal, podendo levar à hemorragia ulcerativa. ${ }^{2}$ Desta forma, a obtenção de inibidores seletivos para a COX-2 pode contornar ou minimizar esses efeitos colaterais.

Por outro lado, substâncias oriundas da catálise da enzima 5-Lipooxigenase (5-LO), como o leucotrieno $\mathrm{B}_{4}$, também contribuem para a hiperalgesia durante a inflamação, por diminuir os limiares mecânicos e térmicos das Fibras C. ${ }^{3}$

Pelas razões mencionadas acima, substâncias que inibem tanto a COX, quanto a 5-LO, se tornam alvos em química medicinal, visando o combate à dor, causada por processos inflamatórios.

Substâncias que contêm em suas estruturas uma porção hidrazona têm sido relatadas na literatura como inibidores da COX e da 5-LO, apresentando, portanto, pronunciadas atividades analgésica e antiinflamatória. Muitas evidências relatadas na literatura demonstram que o grupamento hidrazona, presente em derivados fenil-hidrazonas, apresenta caráter farmacofórico para a inibição de COX. ${ }^{4}$

Várias aril e acil-hidrazonas são relatadas na literatura, apresentando pronunciadas atividades analgésica e anti-inflamatória, além de

*e-mail: rodolfo@ufpe.br outras atividades, como anticonvulsivante e antimicrobiana. ${ }^{5}$

Recentemente, uma nova reação de cicloadição 1,3-dipolar foi desenvolvida, envolvendo enecarbamatos endocíclicos e $N$-óxidos de nitrila, onde novas 2-isoxazolinas aza-bicíclicas foram obtidas. ${ }^{6}$ Derivados carboxamidas dessas 2-isoxazolinas 1 (Figura 1) apresentaram intensa atividade in vivo, com indícios de atividade no sistema nervoso central e possível atividade analgésica, verificadas nos testes de toxicidade aguda. ${ }^{7}$ Testes específicos de atividade anti-<smiles>[R]c1ccc(C(=O)N2CCCC3C(C(N)=O)=NOC32)cc1</smiles>

1

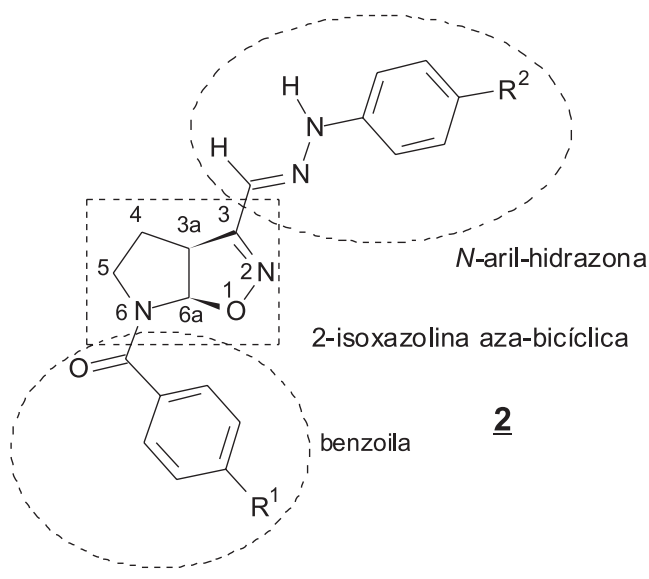

Figura 1. 3-carboxamidas e derivados híbridos hidrazona/2-isoxazolina aza-bicíclica 
inflamatória dessas carboxamidas isoxazolínicas foram realizados, tendo apresentado resultados bastante promissores.

De fato, percebe-se que várias substâncias descritas, que possuem o núcleo 2-isoxazolina, apresentam variadas atividades biológicas, como antimicrobiana, antiviral, antitrombótico, anti-inflamatória, analgésica, entre outras. ${ }^{8}$

A conjugação de características estruturais definidas de dois compostos bioativos distintos, em uma nova molécula, representa a estratégia de hibridização molecular de um composto protótipo. ${ }^{9}$ Portanto, o objetivo deste trabalho foi a síntese de novos derivados híbridos, 2-isoxazolil-aril-hidrazonas $\underline{\mathbf{2}}$ (Figura 1), contendo o núcleo pirrolidina[3,2- $d]$ 2-isoxazolina e a função hidrazona, visando a avaliação prévia da atividade antinociceptiva dos mesmos, com perspectivas posteriores de testes mais específicos para se averiguar um possível mecanismo de ação dos novos fármacos, quanto à seletividade para as enzimas COX-1 e COX-2 ou inibidores duais LO/COX.

\section{RESULTADOS E DISCUSSÃO}

\section{Parte química}

Os aldeídos isoxazolínicos $\underline{\mathbf{8}}$, necessários para a condensação com as fenil-hidrazinas para-substituídas, foram obtidos a partir do cicloaduto $\underline{\mathbf{4}}$, originado de uma reação de cicloadição 1,3-dipolar do enecarbamato endocíclico $\underline{3}$ com o óxido de carboetoxiformonitrila (CEFNO) ${ }^{10,11}$ Esta reação de ciclodição foi desenvolvida em trabalhos anteriores, sendo até aquele momento inédita na literatura. ${ }^{6} \mathrm{~A} 2$-isoxazolina aza-bicíclica racêmica $\underline{4}$ foi obtida em excelente rendimento, de forma régio e diastereoespecífica, conforme previsto pela teoria de orbitais de fronteira. ${ }^{12}$

A posterior reação de hidrogenólise do cicloaduto isoxazolínico $\underline{4}$ propiciou a obtenção da 2 -isoxazolina desprotegida $\underline{\mathbf{5}}$ de forma quantitativa. Em seguida, $N$-benzoilação em N6, com cloretos de benzoíla para-substituídos, levou aos respectivos aza-biciclos $N$-benzoilados $\underline{7}$ em ótimos rendimentos (Esquema 1). ${ }^{6,13}$

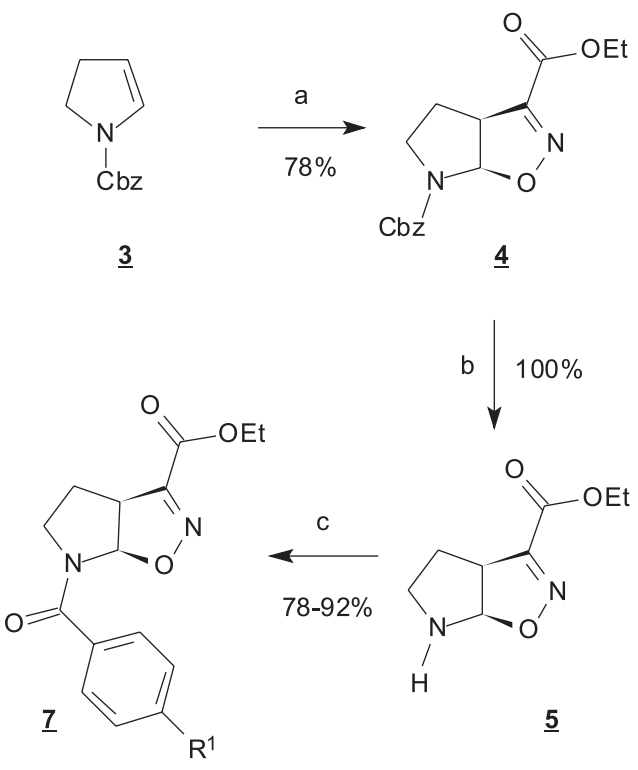

Esquema 1. Rota sintética para obtenção das N-benzoil-isoxazolinas 7 ; a) cloro-oxi-imidoacetato de etila, $\mathrm{Et}_{3} \mathrm{~N}, \mathrm{CHCl}_{3}$, t.a.; b) $\mathrm{H}_{2}, \mathrm{Pd}^{0} / \mathrm{C}$, metanol; c) cloretos de benzoíla, $\mathrm{Et}_{3} \mathrm{~N}, \mathrm{CHCl}_{3}$

Tentativas de redução direta do éster, em C3, dos derivados $\underline{\mathbf{7}}$, aos respectivos aldeídos, não foram bem sucedidas. Ao se utilizar DIBAL-H e super-hidreto como redutores, os ésteres em C3 perma- neceram intactos nas condições reacionais convencionais empregadas. Redutores mais fortes poderiam reduzir também as amidas em N6. O problema na obtenção direta dos aldeídos $\underline{\mathbf{8}}$ foi contornado, reduzindose os ésteres em $\mathrm{C} 3$ aos respectivos alcoóis, com posterior oxidação branda de Swern. ${ }^{14}$ A redução do éster em $\mathrm{C} 3$ com $\mathrm{NaBH}_{4}$ foi bem sucedida, não interferindo na carbonila amídica em N6, resultando nos respectivos alcoóis isoxazolínicos $\underline{\mathbf{6}}$. Dependendo da qualidade do $\mathrm{NaBH}_{4}$ utilizado, percebeu-se primeiramente uma transesterificação, onde ésteres metílicos em $\mathrm{C} 3$ foram obtidos, visto que metanol fora utilizado como solvente nas reações com $\mathrm{NaBH}_{4}$. Este fenômeno provavelmente é devido à catálise promovida por traços de ácidos de Lewis (boranos) presentes no $\mathrm{NaBH}_{4}$. Ao se utilizar $\mathrm{NaBH}_{4}$ de boa procedência, os produtos de transesterificação não foram detectados, o que favoreceu a obtenção direta dos respectivos alcoóis ‥ Após oxidação dos alcoóis ㅁ, via oxidação de Swern, os aldeídos isoxazolínicos em C3 foram obtidos em ótimos rendimentos.

De posse dos aldeídos $\underline{\mathbf{8}}$, procedeu-se à etapa de condensação dos mesmos com as fenil-hidrazinas para-substituídas. ${ }^{3,15}$ As isoxazolil-aril-hidrazonas $\underline{\mathbf{2}}$ foram obtidas em rendimentos moderados, devido principalmente à dificuldade de isolamento das mesmas das águas-mãe, apesar de ter havido total consumo dos aldeídos no meio reacional (Esquema 2). A Tabela 1 lista os rendimentos de todas as reações novas executadas.
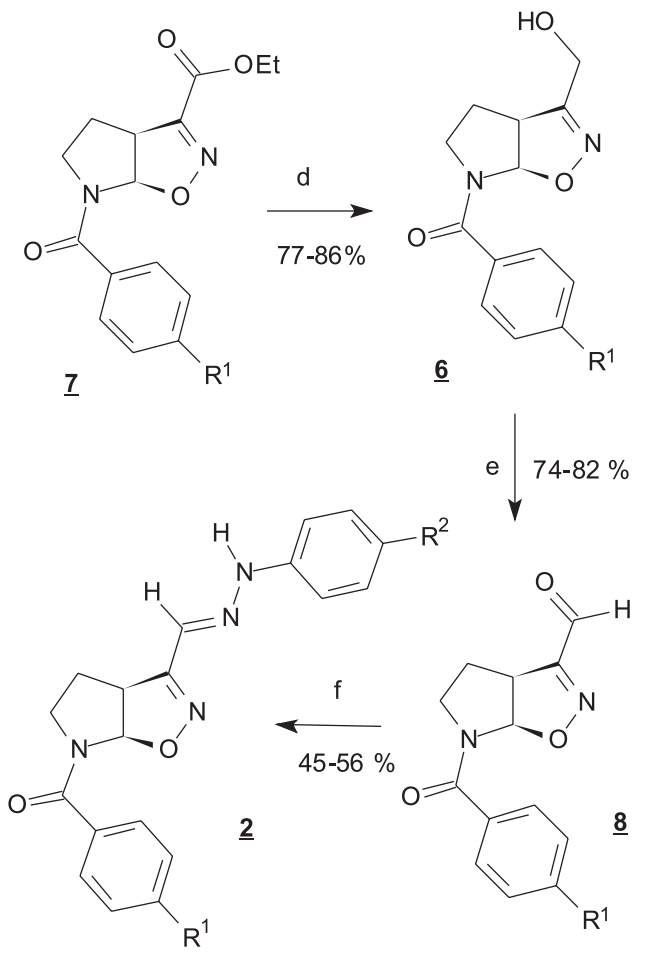

Esquema 2. Rota sintética para obtenção das isoxazolil-aril-hidrazonas 2; d) $\mathrm{NaBH}_{4}$, metanol; e) $\mathrm{SOCl}_{2}$, $\mathrm{DMSO}, \mathrm{Et}_{3} \mathrm{~N}$. $\mathrm{CH}_{2} \mathrm{Cl}_{2}$; f) fenil-hidrazinas, $\mathrm{HCl}_{\text {cat }}$, etanol

A caracterização do biciclo pirrolidina[3,2- $d]$-2-isoxazolina, presente no cicloaduto $\underline{\mathbf{4}}$ e também em $\underline{\mathbf{2}}, \underline{\mathbf{5}}, \underline{\mathbf{6}}, \underline{\mathbf{7}}$ e $\underline{\mathbf{8}}$, se faz facilmente através de espectrometria de RMN, nas quais sinais diagnósticos da formação do cicloaduto são evidentes, como um dubleto próximo a 6,5 ppm em $\mathrm{RMN}{ }^{1} \mathrm{H}$, referente ao H6a e um sinal próximo a $95 \mathrm{ppm}$ em RMN ${ }^{13} \mathrm{C}$, referente ao C6a. Com exceção da isoxazolina desprotegida $\underline{\mathbf{5}}$, todos os derivados isoxazolínicos $\underline{4}, \underline{\mathbf{6}}, \underline{\mathbf{7}}, \underline{\mathbf{8}}$ e $\underline{\mathbf{2}}$ possuem uma função amida ou carbamato em N6. Este fato fez com que os mesmos apresentassem sinais de rotâmeros em RMN de ${ }^{1} \mathrm{H}$ e de ${ }^{13} \mathrm{C}$. A presença de rotâmeros, devido à alta barreira rotacional energética 
Tabela 1. Identificação e rendimentos das novas substâncias 2-isoxazolínicas sintetizadas

\begin{tabular}{cccc}
\hline Substância & $\mathrm{R}^{1}$ & $\mathrm{R}^{2}$ & Rendimento (\%) \\
\hline$\underline{\mathbf{2 a}}$ & $\mathrm{Cl}$ & $\mathrm{Cl}$ & 54 \\
$\underline{\mathbf{2 b}}$ & $\mathrm{Cl}$ & $\mathrm{F}$ & 52 \\
$\underline{\mathbf{2 c}}$ & $\mathrm{Cl}$ & $\mathrm{OMe}$ & 49 \\
$\underline{\mathbf{2 d}}$ & $\mathrm{F}$ & $\mathrm{Cl}$ & 56 \\
$\underline{\mathbf{2 e}}$ & $\mathrm{F}$ & $\mathrm{F}$ & 53 \\
$\underline{\mathbf{2}}$ & $\mathrm{F}$ & $\mathrm{OMe}$ & 40 \\
$\underline{\mathbf{6 a}}$ & $\mathrm{F}$ & -- & 86 \\
$\underline{\mathbf{6 b}}$ & $\mathrm{Cl}$ & -- & 77 \\
$\underline{\mathbf{7 a}}$ & $\mathrm{F}$ & -- & 78 \\
$\underline{\mathbf{7 b}}$ & $\mathrm{Cl}$ & -- & 92 \\
$\underline{\mathbf{8 a}}$ & $\mathrm{F}$ & -- & 74 \\
$\underline{\mathbf{8 b}}$ & $\mathrm{Cl}$ & -- & 82 \\
\hline
\end{tabular}

das ligações amídicas e carbamídicas, deixa os espectros de RMN complexos. ${ }^{16}$ No entanto, quando os espectros foram obtidos à 60 ${ }^{\circ} \mathrm{C}$, ocorreu total coalescência dos sinais de rotâmeros. Outra peculiaridade dos espectros de RMN foi a deformação e alargamento do sinal de H6, assim como de outros hidrogênios exocíclicos do biciclo e também baixa resolução dos sinais dos carbonos do anel bicíclico.

Os hidrogênios diastereotópicos do grupamento hidroximetila dos alcoóis $\underline{6}$ apresentaram-se como dubletos em RMN ${ }^{1} \mathrm{H}$ em 4,35 e 4,45 ppm, respectivamente, com uma constante de acoplamento de 14 $\mathrm{Hz}$, enquanto que o carbono metilênico da hidroximetila dos alcoóis apresentou um deslocamento químico próximo de $55 \mathrm{ppm}$. Os alcoóis 6 também apresentaram nos espectros de IV bandas características em torno de $3505 \mathrm{~cm}^{-1}$, relativamente finas e intensas, caracterizando o estiramento da ligação $\mathrm{O}-\mathrm{H}$ do grupamento hidroximetila em $\mathrm{C} 3$, além do desaparecimento da banda intensa em $1715 \mathrm{~cm}^{-1}$, referente à carbonila do éster em $\mathrm{C} 3$.

A oxidação de Swern dos alcoóis $\underline{\mathbf{6}}$ foi muito bem sucedida e os respectivos aldeídos $\underline{\mathbf{8}}$ foram caracterizados, principalmente pelos sinais em 9,8 e 185 ppm nos espectros de $\mathrm{RMN}{ }^{1} \mathrm{H}$ e ${ }^{13} \mathrm{C}$, respectivamente. Em relação aos espectros no IV, os aldeídos $\underline{\mathbf{8}}$ apresentaram uma banda intensa em $1710 \mathrm{~cm}^{-1}$, característica do estiramento da carbonila do aldeído, que não havia no álcool de partida.

As reações de condensação dos aldeídos $\underline{\mathbf{8}}$ com as fenil-hidrazinas para-substituídas proporcionaram a formação de um único produto. Muito provavelmente, apenas a hidrazona diastereoisomérica de configuração $E$ tenha sido formada, devido a questões cinéticotermodinâmicas. Como isoxazolil-hidrazonas ainda não foram descritas na literatura, não há como fazer analogia de deslocamentos químicos dos hidrogênios $\mathrm{HC}=\mathrm{N}$ de outras hidrazonas, que porventura já possuíssem sua estereoquímica relativa determinada, visto que se tem uma peculiaridade estrutural, pois a função hidrazona, nas moléculas sintetizadas, pertence a uma espécie de heterodieno associado à isoxazolina. Outro fato é que os hidrogênios $\mathrm{HC}=\mathrm{N}$ têm seus sinais geralmente coincidentes com os sinais dos hidrogênios aromáticos, sendo detectada a presença dos mesmos pela integração dos sinais. Diante desses fatos, se faz necessária a obtenção de cristais das hidrazonas, para a obtenção da difração de raio-X das mesmas, visando a confirmação da estereoquímica relativa. No entanto, todas as hidrazonas isoxazolínicas $\underline{\mathbf{2}}$ apresentaram um singleto próximo à 11 ppm em RMN ${ }^{1} \mathrm{H}$, correspondendo ao hidrogênio NN-H da função hidrazona, conforme deslocamentos químicos descritos na literatura para NN-H de outras hidrazonas. ${ }^{3}$ Com relação aos espectros no IV, bandas finas e relativamente intensas, correspondentes ao estiramento NN-H, estão presentes entre $3240-3270 \mathrm{~cm}^{-1}$ confirmando, assim, a função hidrazona nas estruturas dos híbridos $\underline{\mathbf{2}}$. Todos os intermediários inéditos, alcoóis $\underline{\mathbf{6}}$, aldeídos $\underline{\mathbf{8}}$, assim como as hidrazonas $\underline{\mathbf{2}}$, apresentaram os respectivos picos $\mathrm{M}^{+}+1$, com alta resolução, nos espectros de massas obtidos por LCMS-IT/TOF, tendo como fonte de ionização electrospray (ESI).

\section{Parte biológica}

No que concerne à avaliação biológica, as isoxazolil-hidrazonas $\underline{\mathbf{2} \mathbf{a}}$ e $\underline{\mathbf{2 d}}$ foram submetidas preliminarmente ao estudo de toxicidade aguda. ${ }^{17}$ Todos os aspectos reacionais de uma substância no organismo vivo devem ser considerados, quanto aos sinais e sintomas de intoxicação e o percentual de mortalidade para o cálculo de doses letais. Estes valores são calculados estatisticamente a partir de dados obtidos experimentalmente, correlacionando as doses das substâncias e a mortalidade dos animais.

As hidrazonas 2a e 2d revelaram-se de baixa toxicidade na dose de $1500 \mu \mathrm{mol} / \mathrm{kg}$. Foram observados alguns efeitos depressores do sistema nervoso central, comuns a todos os grupos de animais, como ptose, sonolência, contorções abdominais, abaixamento do trem posterior e diminuição da frequência respiratória. No entanto, nenhum animal veio a óbito na dose testada após o período determinado de observação, não sendo possível, portanto, a determinação da $\mathrm{DL}_{50}$. Desta maneira, pode-se concluir que as novas isoxazolil-hidrazonas possuem baixa toxicidade, quando testadas na dose dez vezes superior à dose que apresentou uma excelente atividade terapêutica (atividade antinociceptiva).

Com relação à atividade antinociceptiva, as isoxazolil-hidrazonas 2a-f, administradas por via intraperitoneal, na dose de $150 \mu \mathrm{mol} / \mathrm{kg}$, inibiram significativamente as contorções abdominais induzidas por ácido acético em relação ao controle (Tabela 2). ${ }^{17,18}$

Tabela 2. Percentual de inibição das hidrazonas no teste de contorções abdominais induzidas por ácido acético

\begin{tabular}{ccc}
\hline $\begin{array}{c}\text { Isoxazolil-hidrazonas } \\
(150 \mu \mathrm{mol} / \mathrm{kg})\end{array}$ & $\begin{array}{c}\text { Inibição } \\
(\%)\end{array}$ & $\begin{array}{c}\text { Médias das contorções } \\
( \pm \text { E.P. })^{*}\end{array}$ \\
\hline$\underline{\mathbf{2 a}}$ & 85,8 & $9,8 \pm 1,4$ \\
$\underline{\mathbf{2 b}}$ & 69,6 & $21,0 \pm 2,2$ \\
$\underline{\mathbf{2 c}}$ & 72,0 & $19,2 \pm 1,3$ \\
$\underline{\mathbf{2 d}}$ & 69,3 & $21,2 \pm 3,0$ \\
$\underline{\mathbf{2 e}}$ & 72,1 & $19,2 \pm 1,9$ \\
$\underline{\mathbf{2 f}}$ & 76,0 & $16,4 \pm 2,5$ \\
Dipirona (padrão) & 59,3 & $28,1 \pm 2,8$ \\
Controle (salina) & ---- & $69,1 \pm 2,1$ \\
\hline
\end{tabular}

*Resultados significativos para o nível de $\mathrm{p}<0,05$.

Os fármacos que produzem efeitos analgésicos podem atuar em nível periférico e central, seja modulando ou inibindo a síntese de substâncias mediadoras da inflamação.

A algesia induzida por administração intraperitoneal de ácido acético em camundongos é um modelo de efeito periférico e não específico, envolvendo a liberação de prostaglandinas, serotonina, bradicinina, substância $\mathrm{P}$ e mediadores do sistema simpático. ${ }^{19}$

Os percentuais de inibição foram calculados através da fórmula: Percentual de inibição $=(1-\mathrm{Vt} / \mathrm{Vc}) \times 100$, onde Vt e Vc representam, respectivamente, a média das contorções dos grupos tratados de camundongos e a do controle. Os percentuais de inibição, assim como as médias das contorções resultantes da administração das isoxazolilhidrazonas testadas, estão sumarizados na Tabela 2. 
Os resultados de atividade antinociceptiva mostraram que na dose de $150 \mu \mathrm{mol} / \mathrm{kg}$ as isoxazolil-hidrazonas $\underline{\mathbf{2} \mathbf{a}-\mathbf{f}}$ apresentaram excelentes resultados quando comparados à dipirona, que foi utilizada como fármaco padrão. A inibição das contorções abdominais propiciadas pelas isoxazolil-hidrazonas $\underline{\mathbf{2} \text { a-f }}$ é sugestiva do bloqueio da reação dolorosa por mecanismo periférico, através da inibição da liberação de prostaglandinas e mediadores do simpático. Essas considerações, embora pertinentes, não permitem atribuir o mecanismo exato de atividade antinociceptiva das substâncias testadas, mas indicam uma possibilidade de estudos posteriores, onde testes específicos serão realizados, para aprofundamento acerca do mecanismo de ação.

\section{PARTE EXPERIMENTAL}

\section{Parte química}

As reações de síntese do enecarbamato, a de cicloadição 1,3-dipolar, as de formação dos alcoóis e as de formação dos aldeídos são extremamente sensíveis à umidade e foram realizadas sob atmosfera de argônio, em aparato previamente seco em estufa por $4 \mathrm{~h}\left(120^{\circ} \mathrm{C}\right)$. As reações de síntese das hidrazonas não tiveram essas precauções em relação à umidade.

Clorofórmio foi tratado previamente com cloreto de cálcio anidro e trietilamina foi tratada com hidreto de cálcio e destilados antes do uso. Metanol foi seco com $\mathrm{Mg}^{\circ} / \mathrm{I}_{2}$. THF foi tratado com sódio/ benzofenona e destilado imediatamente antes do uso. DMSO foi seco sobre peneira molecular $4 \AA$, previamente seca em estufa por 24 h. Etanol absoluto foi utilizado nas reações de condensação dos aldeídos com as fenil-hidrazinas, sem necessidade de pré-secagem.

As reações de hidrogenólise foram realizadas sob pressão positiva de hidrogênio, borbulhando-se o gás no meio reacional por cerca de $1 \mathrm{~h}$.

Acetato de etila e hexano, utilizados nas cromatografias em coluna, foram bidestilados.

A análise por cromatografia em camada delgada (CCD) foi efetuada em cromatofolhas de alumínio (gel de sílica 60), com espessura de $0,2 \mathrm{~mm}$, contendo indicador para $254 \mathrm{~nm}$. A visualização das manchas foi efetuada com lâmpada de UV ENF - $2400 \mathrm{C} / \mathrm{F}$ e/ ou ácido fosfomolíbdico $7 \%$ em etanol, seguido de aquecimento ou, ainda, por absorção de iodo.

As colunas cromatográficas foram empacotadas com gel de sílica 60 (230-400 mesh) e eluídas sob pressão (Flash).

Os produtos sólidos tiveram seus pontos de fusão aferidos em aparelhos Polax WRS-1 e não foram corrigidos.

Os espectros no infravermelho (IV) foram obtidos em aparelhos Bruker IFS 66 (transformada de Fourier), com as frequências de absorção sendo expressas em $\mathrm{cm}^{-1}$, utilizando-se pastilhas ou janelas de $\mathrm{KBr}$.

Os espectros de $\mathrm{RMN}{ }^{1} \mathrm{He} \mathrm{e}^{13} \mathrm{C}$ foram obtidos em aparelhos Varian Unity $300 \mathrm{MHz}$. Os deslocamentos químicos ( $\delta$ ) são expressos em ppm, tendo como padrão interno o tetrametilsilano ou $\mathrm{CHCl}_{3}$ para $\mathrm{RMN}{ }^{1} \mathrm{H}$ e DMSO ou $\mathrm{CDCl}_{3}$ para $\mathrm{RMN}{ }^{13} \mathrm{C}$. Os sinais dos espectros de $\mathrm{RMN}{ }^{1} \mathrm{H}$ são designados da seguinte forma: deslocamento químico ( $\mathrm{ppm}$ ), multiplicidade ( $\mathrm{s}=$ singleto, $\mathrm{d}=$ dubleto, $\mathrm{dl}=$ dubleto largo, $\mathrm{t}=$ tripleto, $\mathrm{tl}=$ tripleto largo, $\mathrm{q}=$ quarteto, $\mathrm{dd}=$ duplo dubleto, $\mathrm{m}$ $=$ multipleto, $\mathrm{sl}=$ sinal largo $)$, constante de acoplamento $(\mathrm{Hz}) \mathrm{e}$ números de hidrogênios.

Os espectros de massas de alta resolução (HRMS) foram obtidos em aparelho Shimadzu LCMS IT/TOF, bomba LC 20AD, injetor Sil20 A, tendo como fonte de ionização electrospray (ESI).

\section{Procedimento experimental para a redução do éster. Síntese do álcool $\underline{6 a}$}

A uma solução do éster $\underline{7 \mathbf{a}}(3,767 \mathrm{~g} ; 12,30 \mathrm{mmol})$ em metanol
(180 mL), a $0{ }^{\circ} \mathrm{C}$ e vigorosa agitação, adicionou-se $\mathrm{NaBH}_{4}(1,397$ g; $36,92 \mathrm{mmol}$ ) em pequenas porções. Após a adição, deixou-se em agitação por cerca de $1 \mathrm{~h}$ à $0{ }^{\circ} \mathrm{C}$ e depois 40 min à t.a. A reação foi monitorada por $\mathrm{CCD}$, onde se visualizou a formação do álcool desde o início da adição do $\mathrm{NaBH}_{4}$. Após completo consumo do éster $\underline{\mathbf{7 a}}$, remoção do metanol e solubilização do produto em $50 \mathrm{~mL}$ de acetato de etila, foram adicionados $30 \mathrm{~mL}$ de solução saturada de $\mathrm{NaCl}$, procedendo-se à extração com acetato de etila $(3 \times 40 \mathrm{~mL})$. As fases orgânicas foram combinadas e secas sobre $\mathrm{MgSO}_{4}$ anidro. Após filtração e remoção do solvente, obteve-se um óleo incolor bastante viscoso, que, após ser submetido à cromatografia em coluna flash (gel de sílica, AcOEt/hexano 7:3), foram obtidos 2,600 $\mathrm{g}$ do respectivo álcool puro 6a, com um rendimento de $86 \% ; \mathrm{Rf}=0,1$ (acetato de etila/ hexano 1:1); RMN ${ }^{1} \mathrm{H}$ (DMSO- $d_{6} \delta$, ppm, $300 \mathrm{MHz}$, t.a.), presença de rotâmeros: 2,06 (m, 1H); 2,27 (dd; $J=12,6 ; J=5,7 \mathrm{~Hz} ; 1 \mathrm{H}) ; 3,00$ $(\mathrm{m}, 1 \mathrm{H})$ rotâmero em 3,40; 4,00 à 4,20 $(\mathrm{m}, 2 \mathrm{H}) ; 4,24(\mathrm{~m}, 2 \mathrm{H}) ; 5,39(\mathrm{t}$; $J=6,0 ; 1 \mathrm{H} \mathrm{OH}) ; 6,03$ (d; $J=7,5 ; 1 \mathrm{H})$; rotâmero em 6,53; 7,33 (m; $2 \mathrm{H}) ; 7,67$ (m; $2 \mathrm{H})$; RMN ${ }^{13} \mathrm{C}$ (DMSO- $d_{6} \delta, \mathrm{ppm}, 75 \mathrm{MHz}$, t.a.): 25,9 $\left(\mathrm{CH}_{2}\right)$ rotâmero em 27,$3 ; 43,5\left(\mathrm{CH}_{2}\right)$ rotâmero em 47,4; 52,8 $(\mathrm{CH})$ rotâmero em 50,8; 55,2 $\left(\mathrm{CH}_{2}\right) ; 92,7(\mathrm{CH})$ rotâmero em 90,1; 115,3 $(\mathrm{d} ; J=21 ; \mathrm{CH}) ; 130,3(\mathrm{CH}) ; 132,1(\mathrm{C}) ; 159,9(\mathrm{C}=\mathrm{N}) ; 164,7$ e 161,5 (d; $J=240, \mathrm{C}) ; 167,9(\mathrm{C}=\mathrm{O})$; IV (janela de $\mathrm{KBr}, \mathrm{cm}^{-1}$ ), principais sinais: 3506, 3052, 2970, 2884, 1639, 1597, 1500, 1415, 1210, 1153, 845; HRMS-ESI-IT/TOF $(\mathrm{m} / \mathrm{z}): \mathrm{M}^{+}+\mathrm{H}$, calc. para $\mathrm{C}_{13} \mathrm{H}_{13} \mathrm{FN}_{2} \mathrm{O}_{3}$ : 265,0988; encontrado: 265,0990 6b: óleo incolor, 77\% rendimento, $\mathrm{Rf}=0,11$ (acetato de etila/hexano 1:1); $\mathrm{RMN}^{1} \mathrm{H}\left(\mathrm{CDCl}_{3} \delta, \mathrm{ppm}, 300\right.$ $\mathrm{MHz}$, t.a.), presença de rotâmeros: 2,00 à 2,20 (m, 1H); 2,30 (dd, $J$ $=13 ; J=5,7 ; 1 \mathrm{H}) ; 2,90$ à $3,65(\mathrm{~m}, 2 \mathrm{H}) ; 4,00(\mathrm{~m}, 1 \mathrm{H}) ; 4,35(\mathrm{~d} ; J=$ $14,1 ; 1 \mathrm{H}) ; 4,44(\mathrm{~d} ; J=14,1 ; 1 \mathrm{H}) ; 5,97(\mathrm{~d} ; J=7,0 ; 1 \mathrm{H})$ rotâmero em 6,$72 ; 7,40(\mathrm{~d} ; J=8,1 ; 2 \mathrm{H}) ; 7,64(\mathrm{dl} ; J=8,1 ; 2 \mathrm{H}) . \mathrm{RMN}{ }^{13} \mathrm{C}\left(\mathrm{CDCl}_{3}\right.$, $\delta$, ppm, $75 \mathrm{MHz}$, t.a. $): 26,7\left(\mathrm{CH}_{2}\right) ; 43,4\left(\mathrm{CH}_{2}\right)$ rotâmero em 47,0; 52,6 $(\mathrm{CH})$; 56,8 $\left(\mathrm{CH}_{2}\right)$; 93,3 $(\mathrm{CH})$ rotâmero em 91,0; 128,6 $(\mathrm{CH})$; 129,6 $(\mathrm{CH}) ; 133,3(\mathrm{C}) ; 136,9(\mathrm{C}) ; 158,9(\mathrm{C}=\mathrm{N}) ; 169,1(\mathrm{C}=\mathrm{O})$; IV (janela de $\mathrm{KBr}, \mathrm{cm}^{-1}$ ), principais sinais: 3508, 3080, 2970, 2888, 1632, 1590, 1505, 1420, 1215, 848. HRMS-ESI-IT/TOF $(\mathrm{m} / \mathrm{z}): \mathrm{M}^{+}+\mathrm{H}$, calcd. para $\mathrm{C}_{13} \mathrm{H}_{13} \mathrm{ClN}_{2} \mathrm{O}_{3}$ : 281,0693; encontrado: 281,0703.

\section{Procedimento experimental para a oxidação de Swern. Síntese do aldeído $\underline{\mathbf{8 b}}$}

Foram adicionados a um balão, 1,2 $\mathrm{mL}$ de cloreto de oxalila $(14,18 \mathrm{mmol})$ e $26 \mathrm{~mL}$ de $\mathrm{CH}_{2} \mathrm{Cl}_{2}$ seco e deixou-se sob agitação e atmosfera de argônio à $-60^{\circ} \mathrm{C}$. Paralelamente, foram preparadas duas soluções; a primeira (solução 1) contendo $2 \mathrm{~mL}$ de DMSO seco (28,15 mmol) em $6 \mathrm{~mL}$ de $\mathrm{CH}_{2} \mathrm{Cl}_{2}$ e a segunda (solução 2) contendo o álcool $\underline{\mathbf{6 b}}(3,172 \mathrm{~g} ; 11,3 \mathrm{mmol})$ em $7 \mathrm{~mL}$ de $\mathrm{CH}_{2} \mathrm{Cl}_{2}$. A solução 1 foi adicionada ao balão reacional, permanecendo em agitação por 2 min. Em seguida, a solução 2 foi adicionada lentamente ao balão reacional durante $5 \mathrm{~min}$, permanecendo em agitação por $15 \mathrm{~min}$ adicionais. Em seguida, adicionou-se a trietilamina $(8 \mathrm{~mL}, 57,50$ $\mathrm{mmol}$ ), deixando-se a reação em agitação por $5 \mathrm{~min}$. Através de CCD verificou-se que todo o álcool havia sido consumido, evidenciandose a formação do aldeído $\underline{\mathbf{8 b}}$. Após remoção do solvente, o produto bruto foi submetido à cromatografia em coluna flash (gel de sílica AcOET/hexano 1:4), no qual foram obtidos 2,350 g do aldeído $\underline{\mathbf{8 b}}$ puro, correspondendo a um rendimento de $74 \%$. Sólido amorfo amarelo, $\mathrm{Rf}=0,43$ (acetato de etila/hexano 2:3), p.f $=117-119{ }^{\circ} \mathrm{C}$. RMN ${ }^{1} \mathrm{H}$ (DMSO- $d_{6} \delta, \mathrm{ppm}, 300 \mathrm{MHz}$, t.a.), presença de rotâmeros: 2,00 a 2,40 (m, 2H); 3,07 (m, 1H) rotâmero em 3,94; 4,16 (m, 2H); $6,30(\mathrm{~d} ; J=6,6 ; 1 \mathrm{H})$ rotâmero em 6,$81 ; 7,61(\mathrm{sl} ; 4 \mathrm{H}) ; 9,81(\mathrm{~s}, 1 \mathrm{H})$. RMN ${ }^{13} \mathrm{C}$ (DMSO- $d_{6} \delta$, ppm, $75 \mathrm{MHz}$, t.a.): $26,2\left(\mathrm{CH}_{2}\right)$ rotâmero em 27,6; 43,6 $\left(\mathrm{CH}_{2}\right)$ rotâmero em 46,7; 48,6 $(\mathrm{CH})$ rotâmero em 47,3; 96,9 $(\mathrm{CH})$ rotâmero em 94,0; 128,6 $(\mathrm{CH}) ; 129,6 ;(\mathrm{CH}) ; 134,0(\mathrm{C}) ; 135,4$ (C); 159,8 $(\mathrm{C}=\mathrm{N}) ; 167,8(\mathrm{C}=\mathrm{O}), 185,0(\mathrm{C}=\mathrm{O})$ : IV (pastilha de $\mathrm{KBr}$, 
$\mathrm{cm}^{-1}$ ), principais sinais: $3030,2980,2861,1709,1626,1580,1398$, 1250, 1090, 915, 843. HRMS-ESI-IT/TOF $(\mathrm{m} / z): \mathbf{M}^{+}+\mathrm{H}$, calcd. para $\mathrm{C}_{13} \mathrm{H}_{11} \mathrm{ClN}_{2} \mathrm{O}_{3}$ : 279,0536; encontrado: 279,0550. 8a: 82\% de rendimento, sólido amorfo amarelo, pf. $=112-115^{\circ} \mathrm{C}, \mathrm{Rf}=0,45$ (acetato de etila/hexano 2:3), $\mathrm{RMN}^{1} \mathrm{H}\left(\mathrm{CDCl}_{3} \delta\right.$, ppm, $300 \mathrm{MHz}$, t.a.), presença de rotâmeros: 2,10-2,20 (m, 2H); 3,13 (sl; 1H) rotâmero em 3,66; 4,04 (t; $J=8,1 ; 1 \mathrm{H}) ; 4,44(\mathrm{sl} ; 1 \mathrm{H}) ; 6,24(\mathrm{sl} ; 1 \mathrm{H}) ; 7,12(\mathrm{t} ; J=8,4 ; 2 \mathrm{H})$; $7,69$ (sl; 2H); 9,88 (s, $1 \mathrm{H})$; $\mathrm{RMN}{ }^{13} \mathrm{C}\left(\mathrm{CDCl}_{3} \delta\right.$, ppm, $75 \mathrm{MHz}$, t.a.): $27,3\left(\mathrm{CH}_{2}\right) ; 43,4\left(\mathrm{CH}_{2}\right) ; 48,4(\mathrm{CH}) ; 97,1$ rotâmero em 94,3; 115,6 (d; $J=21 ; \mathrm{CH}) ; 130,5(\mathrm{CH}) ; 132,0(\mathrm{C}) ; 159,1(\mathrm{C}=\mathrm{N}) ; 162,5$ e $165,8(\mathrm{~d}$; $J=250, \mathrm{C}) ; 168,6(\mathrm{C}=\mathrm{O}) ; 185,2(\mathrm{C}=\mathrm{O})$; IV (pastilha de $\mathrm{KBr}, \mathrm{cm}^{-1}$ ), principais sinais: 3048, 2960, 2857, 1710, 1626, 1590, 1388, 1230, 854. HRMS-ESI-IT/TOF $(\mathrm{m} / \mathrm{z}): \mathrm{M}^{+}+\mathrm{H}$, calcd. para $\mathrm{C}_{13} \mathrm{H}_{11} \mathrm{FN}_{2} \mathrm{O}_{3}$ : 263,0832; encontrado: 263,0847.

\section{Procedimento experimental para a reação de condensação. Síntese da hidrazona $\underline{2 a}$}

Foram adicionados em um balão $20 \mathrm{~mL}$ de etanol absoluto e p-clorofenil-hidrazina $(0,257 \mathrm{~g} ; 1,80 \mathrm{mmol})$. Após completa solubilização, sob agitação e à t.a., foram adicionadas duas gotas de $\mathrm{HCl}$ concentrado e, em seguida, o aldeído $\underline{\mathbf{5 8 a}}(0,500 \mathrm{~g} ; 1,80 \mathrm{mmol})$, solubilizado em $10 \mathrm{~mL}$ de etanol. A reação permaneceu em agitação por cerca de 40 min quando, através da CCD, se detectou total consumo do material de partida e formação da hidrazona 2a. Adicionou-se água gelada ao balão reacional, o que proporcionou intensa precipitação do produto, obtendo-se o sólido bruto após filtração em funil sinterizado. O processo de purificação foi realizado através da solubilização das impurezas em acetato de etila à temperatura de $60{ }^{\circ} \mathrm{C}$ e sucessivas filtrações, obtendo-se 0,390 $\mathrm{g}$ da hidrazona $\underline{\mathbf{2 a}}$, correspondendo a um rendimento de $54 \%$. A hidrazona $2 \mathbf{a}$ foi seca em estufa à $45^{\circ} \mathrm{C}$ durante $24 \mathrm{~h}$. Sólido branco amorfo, P.f. $=227-229{ }^{\circ} \mathrm{C}, \mathrm{Rf}=0,51$ (acetato de etila/hexano 2:3). RMN ${ }^{1} \mathrm{H}\left(\mathrm{DMSO}-d_{6} \delta\right.$, ppm, $300 \mathrm{MHz}$, t.a.), presença de rotâmeros: $2,26(\mathrm{~m}, 1 \mathrm{H}) ; 2,51(\mathrm{~m}, 1 \mathrm{H})$ coincide com sinal do DMSO; 3,07 $(\mathrm{m}, 1 \mathrm{H})$ - rotâmero em 3,40 coincidindo com $\mathrm{H}_{2} \mathrm{O}$ do DMSO; 4,00-4,40 (m, 2H); 6,13 (sl; 1H) - rotâmero em 6,64; 7,03 (sl, 2H); 7,27 (sl, 2H); 7,40-7,80 (m, 5H); 11,00 (s, 1H); RMN ${ }^{13} \mathrm{C}$ (DMSO- $d_{6} \delta$, ppm, $75 \mathrm{MHz}$, t.a.): 27,3 $\left(\mathrm{CH}_{2}\right)$ rotâmero em 28,7; 43,5 $\left(\mathrm{CH}_{2}\right)$ rotâmero em 47,5; $51,4(\mathrm{CH})$ rotâmero em 49,5; 93,7 $(\mathrm{CH})$ rotâmero em 91,$1 ; 113,8(\mathrm{CH}) ; 123,5(\mathrm{CH}) ; 127,1(\mathrm{C}) ; 128,4$ $(\mathrm{CH}) ; 129,0(\mathrm{CH}) ; 129,6(\mathrm{HC}=\mathrm{N}) ; 134,2(\mathrm{C}) ; 135,3(\mathrm{C}) ; 142,9(\mathrm{C})$; $157,8(\mathrm{C}=\mathrm{N}) ; 167,8(\mathrm{C}=\mathrm{O})$. IV (pastilha de $\mathrm{KBr}, \mathrm{cm}^{-1}$ ), principais sinais: 3246, 3095, 3020, 2870, 1628, 1553, 1487, 1424, 1250, 1090, 848. HRMS-ESI-IT/TOF $(\mathrm{m} / \mathrm{z}): \mathrm{M}^{+}+\mathrm{H}$, calcd. para $\mathrm{C}_{19} \mathrm{H}_{16} \mathrm{Cl}_{2} \mathrm{~N}_{4} \mathrm{O}_{2}$ : 403,0728; encontrado: 403,0735. 2 2b: $52 \%$ de rendimento, sólido amorfo amarelo pálido, $\mathrm{Rf}=0,49$ (acetato de etila/hexano 2:3), p.f $=221-222^{\circ} \mathrm{C}, \mathrm{RMN}^{1} \mathrm{H}$ (DMSO- $d_{6} \delta$, ppm, $300 \mathrm{MHz}$, t.a.), presença de rotâmeros: 2,27 (m, 1H); 2,45 (m, 1H) coincide com sinal DMSO; $3,09(\mathrm{~m}, 1 \mathrm{H})$ rotâmero em 3,41 coincidindo com $\mathrm{H}_{2} \mathrm{O}$ do DMSO; 4,00 a 4,40 $(\mathrm{m}, 2 \mathrm{H}) ; 6,13(\mathrm{~d} ; J=6 ; 1 \mathrm{H})$ rotâmero em 6,$64 ; 6,69$ a 7,40 (m, $4 \mathrm{H}) ; 7,45$ a $8,00(\mathrm{~m}, 5 \mathrm{H}) ; 10,92(\mathrm{~s}, 1 \mathrm{H})$. RMN ${ }^{13} \mathrm{C}$ (DMSO- $d_{6,}, \mathrm{ppm}$, $75 \mathrm{MHz}$, t.a.): 27,4 $\left(\mathrm{CH}_{2}\right)$ rotâmero em 28,8; 43,6 $\left(\mathrm{CH}_{2}\right)$ rotâmero em 47,3; 51,5 $(\mathrm{CH})$ rotâmero em 49,7; 93,7 ppm $(\mathrm{CH})$ rotâmero em 91,$1 ; 113,5(\mathrm{CH}) ; 116,0$ e $115,7(\mathrm{~d} ; J=22 ; \mathrm{CH}) ; 126,3(\mathrm{CH})$; 128,5 (CH); 129,7 (HC=N); 134,3 (C); 135,3 (C); 140,7 (C); 158,2 e 155,0 (d; $J=240, \mathrm{C}) ; 157,3(\mathrm{C}=\mathrm{N}) ; 167,9(\mathrm{C}=\mathrm{O})$. IV (pastilha de $\mathrm{KBr}, \mathrm{cm}^{-1}$ ), principais sinais: $3243,3120,3030,2943,1618,1550$, 1497, 1424, 1250, 1209, 1147, 856. HRMS-ESI-IT/TOF $(\mathrm{m} / \mathrm{z}): \mathbf{M}^{+}+$ $\mathrm{H}$, calcd. para $\mathrm{C}_{19} \mathrm{H}_{16} \mathrm{ClFN}_{4} \mathrm{O}_{2}: 387,1024$; encontrado: 387,1029 . $2 \mathrm{c}$ : $49 \%$ de rendimento, sólido amorfo ocre claro, $\mathrm{Rf}=0,44$ (acetato de etila 2:3), p.f. $=190-192{ }^{\circ} \mathrm{C}, \mathrm{RMN}{ }^{1} \mathrm{H}$ DMSO- $d_{\sigma}, \delta$, ppm, $300 \mathrm{MHz}$, t.a.), presença de rotâmeros: 2,26 $(\mathrm{m}, 1 \mathrm{H}) ; 2,45(\mathrm{~m}, 1 \mathrm{H})$ coincide com DMSO; 3,07 $(\mathrm{m}, 1 \mathrm{H})$ rotâmero em 3,40 coincidindo com $\mathrm{H}_{2} \mathrm{O}$ do DMSO; 3,70 (s, 3H); 4,00 a 4,40 (m, 2H); 6,11 (sl, 1H) rotâmero em 6,$61 ; 6,80$ a 7,30 (m, 4H); 7,40 a 8,00 (m, 5H); 10,82 (s, 1H); RMN ${ }^{13} \mathrm{C}\left(\mathrm{DMSO}-d_{6} \delta, \mathrm{ppm}, 75 \mathrm{MHz}\right.$, t.a.): $27,4\left(\mathrm{CH}_{2}\right)$ rotâmero em 28,7; 43,6 $\left(\mathrm{CH}_{2}\right)$ rotâmero em 47,3; $51,7(\mathrm{CH})$ rotâmero em 49,8; $55,2\left(\mathrm{CH}_{3}\right)$; 93,5 $(\mathrm{CH})$ rotâmero em 90,$9 ; 113,5(\mathrm{CH}) ; 114,7(\mathrm{CH})$; 124,8 $(\mathrm{CH}) ; 128,5(\mathrm{CH}) ; 129,6(\mathrm{HC}=\mathrm{N}) ; 134,3(\mathrm{C}) ; 135,2(\mathrm{C}) ; 137,9$ (C); 153,5 (C); 158,1 (C=N); 167,9 (C=O); IV (pastilha de $\mathrm{KBr}$, $\mathrm{cm}^{-1}$ ), principais sinais: $3270,3246,3048,2890,2851,1628,1570$, 1502, 1428, 1229, 853. HRMS-ESI-IT/TOF $(\mathrm{m} / \mathrm{z}): \mathbf{M}^{+}+\mathrm{H}$, calcd. para $\mathrm{C}_{20} \mathrm{H}_{19} \mathrm{ClN}_{4} \mathrm{O}_{3}: 399,1224$; encontrado: 399,1232. 2d: $56 \%$ de rendimento, sólido amorfo branco, p.f. $=232-234^{\circ} \mathrm{C}, \mathrm{Rf}=0,51$ (acetato de etila/hexano 2:3), RMN ${ }^{1} \mathrm{H}$ (DMSO- $d_{6} \delta$, ppm, $300 \mathrm{MHz}$, t.a.), presença de rotâmeros: 2,26 (m, 1H); 2,43 (m, 1H) coincide com sinal DMSO; $3,07(\mathrm{~m}, 1 \mathrm{H})$ rotâmero em 3,46 ppm, coincidindo com $\mathrm{H}_{2} \mathrm{O}$ do DMSO; 4,15 (m, 1H); 4,27 (tl, $J=8,0 ; 1 \mathrm{H}) ; 6,15(\mathrm{~d} ; J=6,3 ; 1 \mathrm{H})$ rotâmero em 6,65; 7,03 (d, $J=8,1 ; 2 \mathrm{H}) ; 7,30(\mathrm{~m}, 5 \mathrm{H}) ; 7,71(\mathrm{sl}, 2 \mathrm{H})$; $11,03$ (s, $1 \mathrm{H}) ; \mathrm{RMN}^{13} \mathrm{C}$ (DMSO- $d_{6} \delta$, ppm, $75 \mathrm{MHz}$, t.a.): $27,4\left(\mathrm{CH}_{2}\right)$ rotâmero em 28,7; 43,6 $\left(\mathrm{CH}_{2}\right)$ rotâmero em 47,3; 51,4 $(\mathrm{CH})$ rotâmero em 49,5; $93,9(\mathrm{CH})$ rotâmero em 91,2; 113,8 $(\mathrm{CH}) ; 115,4$ (d; $J=21$; $\mathrm{CH}) ; 123,5(\mathrm{C}) ; 127,3(\mathrm{CH}) ; 129,1(\mathrm{CH}) ; 130,3(\mathrm{HC}=\mathrm{N}) ; 132,0(\mathrm{C})$; 143,0 (C); 157,9 (C=N); 164,8 e 161,5 (d; $J=247, \mathrm{C}) ; 167,9$ (C=O). IV (pastilha de $\mathrm{KBr}, \mathrm{cm}^{-1}$ ), principais sinais: $3254,3090,3040,2875,1625$, 1600, 1565, 1485, 1247, 1159, 852. HRMS-ESI-IT/TOF ( $/ \mathrm{m} / \mathrm{z}): \mathbf{M}^{+}+\mathrm{H}$, calcd. para $\mathrm{C}_{19} \mathrm{H}_{16} \mathrm{ClFN}_{4} \mathrm{O}_{2}: 387,1024$; encontrado: 387,1034. 2e: $53 \%$ de rendimento, sólido amorfo ocre claro, p.f. $=227-229^{\circ} \mathrm{C}, \mathrm{Rf}=0,46$ (acetato de etila /hexano 2:3); $\mathrm{RMN}{ }^{1} \mathrm{H}\left(\mathrm{DMSO}-d_{6}, \delta\right.$, ppm, $300 \mathrm{MHz}$, t.a.), presença de rotâmeros: 2,26 (m, 1H); 2,40 (m, 1H) coincide com sinal DMSO; 3,08 $(\mathrm{m}, 1 \mathrm{H})$ - rotâmero em 3,43 coincidindo com $\mathrm{H}_{2} \mathrm{O}$ do DMSO; 4,00 a 4,40 (m, 2H); 6,14 (sl, 1H) rotâmero em 6,65; 6,90 a 7,50 (m, 6H); 7,70 (sl, 3H); 10,93 (s, 1H); RMN ${ }^{13} \mathrm{C}\left(\mathrm{DMSO}-d_{6,} \delta\right.$, ppm, $75 \mathrm{MHz}$, t.a.): 27,4 $\left(\mathrm{CH}_{2}\right)$ rotâmero em 28,$7 ; 43,6\left(\mathrm{CH}_{2}\right)$ rotâmero em 47,4; 51,5 $(\mathrm{CH})$ rotâmero em 49,6; 93,8 $(\mathrm{CH})$ rotâmero em 91,5; $113,5(\mathrm{CH}) ; 115,9(\mathrm{~d} ; J=22 ; \mathrm{CH}) ; 115,4(\mathrm{~d} ; J=22 ; \mathrm{CH}) ; 126,4(\mathrm{CH})$; 130,3 (HC=N); 132,0 (C); 140,7 (C); 158,2 e 155,1 (d; $J=232, \mathrm{C})$; $157,8(\mathrm{C}=\mathrm{N}) ; 164,8$ e $161,5(\mathrm{~d} ; J=247$; $) ; 167,7(\mathrm{C}=\mathrm{O})$; IV (pastilha de $\mathrm{KBr}, \mathrm{cm}^{-1}$ ), principais sinais: $3240,3125,3041,2898,1630,1565$, 1503, 1429, 1260, 1210, 1205, 1152, 850. HRMS-ESI-IT/TOF $(\mathrm{m} / \mathrm{z})$ : $\mathrm{M}^{+}+\mathrm{H}$, calcd. para $\mathrm{C}_{19} \mathrm{H}_{16} \mathrm{~F}_{2} \mathrm{~N}_{4} \mathrm{O}_{2}: 371,1319$; encontrado: 371,1325.

\section{Parte biológica}

Todos os resultados foram expressos em média \pm erro padrão da média. No estudo da atividade antinociceptiva utilizou-se análise de variância (ANOVA) e as diferenças foram consideradas significativas quando $\mathrm{p}<0,05$.

\section{Animais}

Foram utilizados camundongos albinos Swiss (Mus musculus), machos, com aproximadamente 60 dias de vida, variando de 25 a 30 g, oriundos do Biotério do Departamento de Antibióticos da Universidade Federal de Pernambuco, registrado no COBEA (Colégio Brasileiro de Experimentação Animal) sob no 18. Os animais foram divididos em grupos e mantidos em caixas plásticas à temperatura 23 $\pm 2{ }^{\circ} \mathrm{C}$ com ciclos claro/escuro de $12 \mathrm{em} 12 \mathrm{~h}$, recebendo ração padrão e água ad libitum, estando em jejum de $4 \mathrm{~h}$ antes dos experimentos.

\section{Toxicidade aguda}

Os camundongos foram separados em grupos de 6 animais. Em seguida, as substâncias $\underline{\mathbf{2} \mathbf{a}}$ e $\underline{\mathbf{2}} \mathbf{d}$ foram administradas por via intraperitoneal na dose de $1500 \mathrm{mg} / \mathrm{kg}$. A observação dos efeitos tóxicos foi efetuada com os animais em livre movimentação, em superfície plana, por um período inicial de $60 \mathrm{~min}$. Após esse período, os animais foram ainda observados durante $24 \mathrm{~h}$ para registro do índice de mortalidade de cada grupo e durante 14 dias para observações gerais. ${ }^{18}$ 


\section{Atividade antinociceptiva}

A atividade antinociceptiva foi verificada através do teste de contorção abdominal induzida pelo ácido acético. Os animais foram arranjados aleatoriamente (6 camundongos por grupo). Cada grupo foi pré-tratado, por via intraperitoneal, com uma das hidrazonas $\underline{\mathbf{2 a}-\mathbf{f}}$ $(150 \mu \mathrm{mol} / \mathrm{kg})$ e a substância padrão dipirona $(150 \mu \mathrm{mol} / \mathrm{kg}), 60 \mathrm{~min}$ antes do teste. O grupo controle recebeu o veículo (salina). Ácido acético $1 \%$ foi injetado dentro da cavidade peritoneal dos animais, para induzir contrações da musculatura abdominal e/ou alongamento dos membros posteriores. Dez minutos após a injeção do ácido acético, os camundongos foram colocados individualmente em caixas transparentes, registrando-se o número de contorções abdominais durante $20 \mathrm{~min}$. A percentagem de inibição foi calculada mediante a redução do número de contorções totais nos grupos pré-tratados em relação ao grupo controle. ${ }^{18}$

\section{CONCLUSÃO}

Neste trabalho, foi explorado química e biologicamente o novo heterobiciclo pirrolidina[3,2-d]-2-isoxazolina, através da aplicação sintética deste núcleo na obtenção de derivados hidrazonas. Novos aldeídos isoxazolínicos, obtidos a partir do cicloaduto da reação de ciclodição 1,3-dipolar entre enecarbamato endocíclico e $N$-óxido de nitrila, foram condensados com fenil-hidrazinas, obtendo-se novas isoxazolil-hidrazonas, que possuem dois sítios distintos de substituição, visando a variação estrutural das mesmas. Rendimentos ótimos foram obtidos nas etapas de síntese dos aldeídos, enquanto que na etapa de condensação entre os aldeídos e as fenil-hidrazinas, rendimentos moderados foram obtidos, devido a problemas no isolamento dos produtos.

As avaliações biológicas preliminares das novas isoxazolilhidrazonas mostraram que as mesmas possuem baixa toxicidade $\mathrm{e}$ excelente atividade antinociceptiva, quando comparadas ao fármaco padrão dipirona. Estes resultados são bastante promissores e corroboram resultados anteriores, relativos à atividade biológica da isoxazolina aza-bicíclica. Diante dos resultados obtidos, a série de isoxazolil-hidrazonas deverá ser expandida, visando uma variação estereoeletrônica das mesmas e testes específicos de atividade analgésica, para uma possível determinação do mecanismo de ação, assim como testes anti-inflamatórios, entre outros, deverão ser realizados.

\section{AGRADECIMENTOS}

Ao CNPq e à FACEPE pelo apoio financeiro e à Central Analítica do DQF-UFPE pelas análises espectrométricas.

\section{REFERÊNCIAS}

1. De Leval, X.; Delarge, J.; Somers, F.; De Tullio, P.; Henrotin, Y.; Pirotte, B.; Dogné, J. M.; Curr. Med. Chem. 2000, 7, 1041; Chandrasekharan, N. V.; Dai, H.; Roos, K. L.; Evanson, N. K.; Tomsik, J.; Elton, T. S.; Simmons, D. L.; Proc. Natl. Acad. Sci.USA 2002, 99, 13926.

2. Dammann, H. G. Z.; Gastroenterology 1999, 37, 45.

3. Lima, P. C.; Lima, L. M.; Da Silva, K. C. M.; Léda, P. H. O.; De Miranda, A. L. P.; Fraga, C. A. M.; Barreiro, E. J.; Eur. J. Med. Chem. 2000, 35, 187.

4. Mahy, J. P.; Gaspard, S.; Mansuy, D.; Biochemistry 1993, 32, 4014.

5. Todeschini, A. R.; De Miranda, A. L. P.; Da Silva, K. C.; Parrini, S. C.; Barreiro, E. J.; Eur. J. Med. Chem. 1998, 33, 189; Küçükgüzel, S. G.; Rollas S.; Il Farmaco 2002, 57, 583; Dimmock, J. R.; Vashishtha, S. C.; Stables, J. P.; Eur. J. Med. Chem. 2000, 35, 241; Çakir, B.; Yildirim, E.; Ercanli, T.; Erol, K.; Sahin, M. F.; Il Farmaco 1999, 54, 842.

6. De Almeida, V. M.; Dos Santos, R. J.; Góes, A. J. S.; De Lima, J. G.; Correia, C. R. D.; De Faria, A. R.; Tetrahedron Lett. 2009, 50, 684.

7. De Almeida, V. M.; Dos Santos, R. J.; Góes, A. J. S.; De Souza, I. A.; De Faria, A. R.; Rev. Bras. Cienc. Saúde 2005, 9, 275.

8. Pirrung, M. C.; Tumey, L. N.; Raetz, C. R. H.; Rusche, K. M.; J. Med. Chem. 2002, 45, 4359; Kai, H.; Matsumoto, H.; Hattori, N.; Takase, A.; Fujiwara, T.; Sugimoto, H.; Bioorg. Med. Chem. Lett. 2001, 11, 1997; Pruitt, J. R.; Pinto, D. J.; Estrella, M. J.; Bostrom, L. L.; Knabb, R. M.; Wong, P. C.; Wright, M. R.; Wexler, R. R.; Bioorg. Med. Chem. Lett. 2000, 10, 685 .

9. Viegas-Junior, C.; Danuello, A.; Bolzani, V. S.; Barreiro, E. J.; Fraga, C. A. M.; Curr. Med. Chem. 2007, 14, 1829; Barreiro, E. J.; Fraga, C. A. M.; Química Medicinal - As Bases Farmacológicas da Ação dos Fármacos, Ed. ArtMed: Porto Alegre, 2001.

10. Kraus, G. A.; Neuenschwander, K.; J. Org. Chem. 1981, 46, 4791

11. Kozikowski, A. P.; Adamczyk, M.; J. Org. Chem. 1983, 48, 366.

12. Fleming, I.; Frontier Orbitals and Organic Chemical Reactions, John Willey and Sons: New York, 1976.

13. De Faria, A. R.; Salvador, E. L.; Correia, C. R. D.; J. Org. Chem. 2002, 67, 3651 .

14. Schmitz, W. D.; Messerschmidt, N. B.; Romo, D.; J. Org. Chem. 1998, 63, 2058; Estieu, K.; Paugam, R.; Ollivier, J.; Salaun, J.; Cordero, F. M.; Goti, A.; Brandi, A.; J. Org. Chem. 1997, 62, 8276.

15. Gaston, M. A.; Dias, L. R. S.; Freitas, A. C. C.; Miranda, A. L. P.; Barreiro, E. J.; Pharm. Acta Helv. 1996, 71, 213.

16. Fontoura, L. A. M.; Rigotti, I. J. C.; Correia, C. R. D.; J. Mol. Struct. 2002, 609, 73

17. Oga, Z.; Avaliação da Toxicidade - Fundamentos de Toxicologia, Ed. Atheneu: São Paulo, 1996; Pérez-Guerrero, C.; Herrera, M. D.; Ortiz, R.; Sotomayor, M. A.; Fernandez, M. A.; J. Ethnopharmacol. 2001, 76, 279.

18. Vane, J. R.; Botting, R. M.; Am. J. Med. 1998, 104, 2S; Cunha, F.Q.; Rev. Bras. Reumatol. 2004, 44, 1; Koster, R.; Anderson, M.; De Beer, E. J.; Fed. Proc. 1959, 18, 412.

19. Duarte, J. D. G.; Negus, S. S.; Butelman, E. R., Costa, B. R.; Woods, J. H. Psychopharmacology 1998, 115, 311. 